\title{
Performance of Multidrug-Resistant Tuberculosis Surveillance in Yemen: Interview Study
}

Jihan Abdulmughni $^{1^{*}}$, MS; Esam Mohammed Mahyoub ${ }^{2 *}, \mathrm{PhD}$; Abdulaziz Thabit Alaghbari ${ }^{2 *}$, MD; Abdulwahed Abdelgabar Al Serouri ${ }^{1 *}, \mathrm{PhD}$; Yousef Khader ${ }^{3 *}, \mathrm{ScD}$

\footnotetext{
${ }^{1}$ Yemen Field Epidemiology Training Program, Sana'a, Yemen

${ }^{2}$ National Tuberculosis Control Program, Sana'a, Yemen

${ }^{3}$ Jordan University of Science and Technology, Amman, Jordan

*all authors contributed equally
}

\section{Corresponding Author:}

Jihan Abdulmughni, MS

Yemen Field Epidemiology Training Program

Ministry of Public Health and Population

Sana'a

Yemen

Phone: 967771299020

Fax: 9671465041

Email: gihanthr@gmail.com

\section{Abstract}

Background: Multidrug-resistant tuberculosis (MDR-TB) is a major challenge to ending TB occurrence by 2035. In Yemen, the 2011 survey showed an MDR-TB prevalence of 1.4\% among new cases and $14.4 \%$ among previously treated cases. The National Tuberculosis Control Program (NTCP) established four MDR-TB sentinel surveillance sites in 2013 to monitor the MDR-TB situation. In Yemen, the 2011 survey showed an MDR-TB prevalence of 1.4\% among new cases and 14.4\% among previously treated cases. The NTCP established four MDR-TB sentinel surveillance sites in 2013 to monitor the MDR-TB situation.

Objective: This study aimed to assess the performance of MDR-TB surveillance and determine its strengths and weaknesses.

Methods: We used the updated Center for Diseases Control and Prevention guidelines for evaluating public health surveillance systems. Interviews were conducted with NTCP managers and Regional MDR-TB centers' staff using a semistructured questionnaire. We used a 5-point Likert scale to assess the usefulness and other attributes (eg, simplicity and flexibility). The mean percentage was calculated for each attribute and used for the final rank of the performance: poor $(<60 \%)$, average $(60 \%-80 \%)$, and $\operatorname{good}(>80 \%)$.

Results: The MDR-TB surveillance system achieved good performance in usefulness (87\%), acceptability (82\%), and data quality (91\%); average performance in flexibility $(61 \%)$ and simplicity $(72 \%)$; and poor performance in stability $(55 \%)$. The overall performance score was average $(74 \%)$. Although strong commitment, good monitoring, and well-trained staff are the main strengths, depending on an external fund is a major weakness along with unavailability of the MDR-TB unit at the governorate level.

Conclusions: Although the MDR-TB surveillance system has achieved an average overall performance, more efforts are required to improve its stability by ensuring constant power supply to enable laboratories to perform necessary diagnostic and follow-up tests. Gradual replacement of donors' funds by the government is recommended. Scaling up of MDR-TB services and removing access barriers are crucial.

(JMIR Public Health Surveill 2019;5(4):e14294) doi: 10.2196/14294

\section{KEYWORDS}

multidrug-resistant tuberculosis; surveillance evaluation; Yemen; field epidemiology program 


\section{Introduction}

Tuberculosis (TB) is one of the major causes of morbidity and mortality worldwide, with more than 10 million newly reported cases and 1.7 million deaths in 2015 [1]. With an estimated 480,000 new multidrug-resistant (MDR) TB cases every year, developing drug resistance to anti-TB drugs becomes a major challenge for the global prospect of ending TB by 2035 [2]. Although MDR-TB is defined as the resistance to rifampicin and isoniazid, extensively drug-resistant TB (XDR-TB) is an MDR-TB with resistance to fluoroquinolone and at least one of the injectable second-line anti-TB drugs [3]. As MDR-TB is more difficult and costly to treat, there are increasing concerns about its continued spread and negative impact on the population and health systems [4]. Effective response to MDR-TB should not only focus on treating drug-susceptible tuberculosis, but also include strong surveillance systems, drug susceptibility testing for all patients with tuberculosis, rapid linkage to effective treatment, and patient-centered care throughout the treatment course [5].

Globally, in 2015, 4\% of all new patients and $20 \%$ of previously treated patients with TB had MDR-TB. China, India, and the Russian Federation account for nearly half of the global MDR-TB prevalence [6]. The data on MDR - TB from eight countries of the Eastern Mediterranean Region (Egypt, Islamic Republic of Iran, Jordan, Lebanon, Morocco, Oman, Qatar, and Yemen) showed an MDR-TB prevalence of 2.0\% among new cases and $35.3 \%$ among previously treated cases [7]. Although most countries in the region had established MDR-TB management in line with World Health Organization (WHO) guidance, it estimated that the region has only detected $12 \%$ of MDR-TB cases and has enrolled $72 \%$ of them on treatment.

In Yemen, TB is the fourth biggest public health problem, with an incidence of 48 per 100,000 people [8]. However, there were no systems in place for the management of MDR in Yemen before 2013, as shown by two surveys performed in 2005 and 2011. The 2004-2005 survey showed an MDR-TB prevalence of $2.9 \%$ among new smear-positive cases and $11.3 \%$ among previously treated cases compared to $1.4 \%$ and $14.4 \%$, respectively, in the 2010-2011 survey [9]. By the end of 2013, the National TB Control Program (NTCP) started the DR-TB Management and Surveillance program. This study aimed to evaluate the performance of MDR-TB surveillance in Yemen and determine its strengths and weaknesses.

\section{Methods}

\section{Evaluation Design}

A descriptive evaluation study was conducted to assess the performance of MDR-TB surveillance system in Yemen using the Updated CDC guideline for evaluation of public health surveillance system [8]. The study was conducted at Sana' a city from November to December 2016. Data on MDR-TB were collected from the NTCP and the four Regional MDR-TB centers.

\section{The Multidrug-Resistant Tuberculosis Surveillance System}

The MDR-TB is a sentinel system through four regional MDR-TB centers at Sana'a City, Aden, Taiz, and Al Hodaidah, with an aim to cover the whole country. The centers provide detection, diagnosis, treatment, and follow-up through a community-based outpatient treatment-delivery strategy that consists of a community supporter, community nurse, drug provider, coordinator, and lab technician. The community supporter is one of the patient's family members who observes the patient daily, has contact with the community nurse regularly, and fills monthly reports for daily treatment intake. The community nurse follows the community supporters on a monthly basis, directly contacts patients during the follow-up visits, and is responsible for registration of DR-TB. The drug provider is a nurse responsible for supplying drugs to patients with MDR-TB and for the drug supply registry. All patients have monthly clinical assessments by a specialist doctor. The lab technician is responsible for performing the smears and cultures or others lab tests in addition to completing the lab registry and reports. The MDR-TB coordinator is responsible for sending the reports to the NTCP. All centers have equipped labs for drug susceptibility testing (DST) and culture. Due to a total breakdown of the electricity network that is needed for DST, the regional MDR-TB centers were provided with the GenXpert, except for the Taiz MDR-TB center, which still sends samples to Sana'a City for diagnosis.

At the MDR-TB centers, there are three hard-copy registers: the DR-TB case register, the lab register, and the MDR-TB drug supply register. The DR-TB cases register contains a set of variables including demographic variables, previous TB registration number, type of drug resistance, starting category 4 treatment date, and use of previous second-line drugs, DST result, smear and culture follow-up results, and HIV status.

\section{Data Collection and Analysis}

The MDR-TB documents were reviewed to describe the system. The mangers of the NTCP were interviewed to assess usefulness, flexibility, and stability of the MDR-TB system. Semistructured questionnaires were used to collect data from staff of the four MDR-TB centers to evaluate simplicity and acceptability of the system. Specific items were used to evaluate each performance attribute. Respondents from the four centers were requested to rate the items measuring the system usefulness as well as the simplicity, flexibility, acceptability, and stability using the 5 -point Likert scale (1=strongly disagree, $2=$ disagree, $3=$ not sure, $4=$ agree, $5=$ strongly agree). The mean percentage score was calculated for each attribute, where a higher score indicates a higher level of performance. The performance was interpreted using the following scoring system that was used in previous similar studies: $<60 \%$, poor; $60 \%-80 \%$, average; and $>80 \%$, good [10-12]. To assess the data quality, we reviewed $10 \%$ of randomly selected MDR-TB cases' files and registers. The selected files were reviewed for any missing data or discrepancies between data on cases' files and register. Epi info version 7.2 (Centers of Disease Control and Prevention, Atlanta, Georgia) was used for data entry and analysis. 


\section{Results}

\section{Data Flow of Multidrug-Resistant Tuberculosis Surveillance}

Figure 1 shows the data flow of the MDR-TB surveillance system, where the suspected case is sent to the nearest regional DR-TB center for lab confirmation and treatment. According to the tests results, the lab sends immediate reports for positive cases to the NTCP to be included in their drug supply for the reporting regional DR-TB. Each regional DR-TB center sends quarterly reports to the NTCP regarding MDR-TB cases during that quarter including lab report, drug supply, and treatment outcome. The NTCP sends quarterly and annual reports to the WHO.

\section{Usefulness}

The NTCP managers agreed that the data are useful. They reported that they used the data to estimate needs of the NTCP in terms of drugs and lab tests. Furthermore, all managers agreed that the MDR-TB data are used to estimate the TB magnitude, incidence, and mortality rates in order to monitor the trend of MDR-TB over time; identify areas at greater risk; update and develop the strategic direction for MDR-TB activity; and plan the resources for detection, prevention, and control activities. The usefulness percentage score was $87 \%$, which indicates that the system is useful (Table 1).

\section{Flexibility}

All NTCP managers agreed that the system is able to adapt any change in case definition. Four managers $(80 \%)$ reported that the system is flexible and can be integrated with other surveillance systems. For example, the system is integrated with the HIV program, where all MDR-TB cases are tested for HIV, and positive cases are reported to the HIV program. The flexibility score of the system was $61 \%$, indicating average flexibility (Table 2).

Figure 1. Data flow of multiple drug-resistant tuberculosis cases in Yemen. WHO: World Health Organization; TB: tuberculosis.

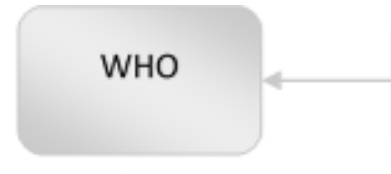

Annual \&quarterly reports

\section{National TB Control Program}

-Immediate report for positive case

-Quarterly report
Center for Drug

Resistance - TB

Suspected case

Table 1. The usefulness of the multidrug-resistant tuberculosis surveillance system as assessed by the five managers of the National Tuberculosis Control Program in Yemen.

\begin{tabular}{|c|c|c|c|}
\hline Statements & Score & Proportion & Rank \\
\hline The MDR-TB ${ }^{\mathrm{a}}$ data are used to estimate the MDR-TB magnitude, incidence, and mortality rates & 4.4 & 88 & Good \\
\hline The data are used to monitor the trend of MDR-TB spread over time & 4.0 & 80 & Good \\
\hline The data are used to identify areas at greater risk & 4.2 & 84 & Good \\
\hline The data are used for planning the resources for detection, prevention, and control activities & 4.6 & 92 & Good \\
\hline The data are used to update and develop the strategic direction for MDR-TB activity & 4.4 & 88 & Good \\
\hline The collected data are used to estimate the drugs and laboratory test needed & 5.0 & 100 & Good \\
\hline The data are used to identify research priorities & 4.0 & 80 & Good \\
\hline Average & 4.4 & 87 & Good \\
\hline
\end{tabular}

${ }^{\mathrm{a}}$ MDR-TB: multidrug-resistant tuberculosis.

Table 2. The flexibility of the multidrug-resistant tuberculosis surveillance system as assessed by the five managers of the National Tuberculosis Control Program in Yemen, 2016.

\begin{tabular}{|c|c|c|c|}
\hline Statements & Score & Proportion & Rank \\
\hline The system can be adapted to accommodate addition/change to case definitions. & 4.0 & 80 & Good \\
\hline The system can be integrated with other surveillance such as HIV & 3.6 & 72 & Good \\
\hline The system is not affected by outside funding & 1.6 & 32 & Poor \\
\hline Average & 3.1 & 61 & Average \\
\hline
\end{tabular}




\section{Stability}

All participants said that unscheduled outages have been rarely occurred before the war. Three $(60 \%)$ managers said that the system would collapse if the donor fund stops. The stability of the system was poor, as evidenced by the stability score of $55 \%$ (Table 3).

\section{Weakness and Strength of the Multidrug-Resistant Tuberculosis Surveillance System}

According to the NTCP managers, the strengths of the system include the program's commitment to provide a treatment for cases, good monitoring system, well-trained staff, and strong donor support. On the other hand, the managers stated that dependence on the external fund is a major weakness of the system, especially if stopped. Other weaknesses included unavailability of the MDR-TB center at the governorate level, absent inpatient department, no refresher training or training for new staff, absence of an electronic system, no trained staff for data analysis at the regional MDR-TB centers levels, and no constant electrical power supply for the lab.

\section{Simplicity}

All the 16 regional MDR-TB staff agreed that the case definitions are easy and clear. Although $71 \%$ reported that the guidelines are easy and understandable, $50 \%$ reported that it is preferable to have these guidelines written in Arabic. Regarding data collection, $44 \%$ respondents reported that this is difficult because, sometimes, the data are needed to call the patient and $66 \%$ reported that the data require regular follow-up. All the respondents agreed that transferring data to the NTCP is easy. However, $56 \%$ of the respondents reported that there is shortage in lab tests, especially the routine follow-up lab tests (eg, liver function, renal function, and eye and hearing tests) that are only available at the Sana'a regional MDR-TB center. The majority (94\%) stated that they received training only once in 2014 , and all stated that they need refresher training. The simplicity attribute has been scored $72 \%$, which means that the system is average in simplicity (Table 4).

Table 3. The stability of the multidrug-resistant tuberculosis surveillance system as assessed by the five managers of the National Tuberculosis Control Program in Yemen.

\begin{tabular}{llll}
\hline Statements & Score & Proportion & Rank \\
\hline Unscheduled outages/electrical power off rarely occur & 1.4 & 28 & 32 \\
You have your own source & 1.6 & 80 & Poor \\
There is planned resources for maintenance of the system & 4.0 & 28 & Poor \\
The system is stable even with cutting foreign fund & 1.4 & 80 & Poor \\
Trained staff rarely turnover & 4.0 & 80 & Good \\
Data release regularly & 4.0 & 55 & Good \\
Average & 2.7 & Poor \\
\hline
\end{tabular}

Table 4. The simplicity of the multidrug-resistant tuberculosis surveillance system as assessed by 16 service providers in Yemen.

\begin{tabular}{|c|c|c|c|}
\hline Statements & Mean score & Percentage score & Rank \\
\hline Case definition is available and easy to apply & 4 & 80 & Good \\
\hline You have easy guide to use & 3.5 & 70 & Average \\
\hline Registers or form is easy to fill & 4 & 80 & Good \\
\hline Collecting case detailed information don't need telephone or visit & 3 & 60 & Poor \\
\hline Collecting data do not need much time & 3 & 60 & Poor \\
\hline You received training on $\mathrm{MDR}^{\mathrm{a}}$ surveillance & 3.8 & 76 & Average \\
\hline Transferring data to high level is very easy & 4 & 80 & Good \\
\hline Data do not need regular follow up & 2.6 & 52 & Poor \\
\hline Laboratory media, solutions and equipment always available & 2.7 & 52 & Poor \\
\hline No shortage in supplying drugs happened & 3.5 & 70 & Average \\
\hline Average & 3.6 & 70 & Average \\
\hline
\end{tabular}

aMR: multidrug resistant. 


\section{Acceptability}

All the respondents indicated that they receive quarterly incentives for their work and that they are willing to continue participating in the MDR-TB surveillance system. Overall, $81 \%$ of the respondents were satisfied. Acceptability scored 82\%, which indicates that the system has good acceptability (Table $5)$.

\section{Data Quality}

By reviewing the regional Sana'a city DR-TB center's registers and cases files, we found that $5 \%$ and $20 \%$ had missing data, respectively. However, comparison of data on case files and register did not show any discrepancies.

\section{Overall Performance of the Multidrug-Resistant Tuberculosis Surveillance System}

The performance of the surveillance system is illustrated in Table 6 .

Table 5. The acceptability of the multidrug-resistant tuberculosis surveillance system as assessed by 16 service providers in Yemen.

\begin{tabular}{llll}
\hline Statements & Mean score & Percentage score & Rank \\
\hline You are willing to participate in MDR-TB ${ }^{\mathrm{a}}$ surveillance & 4.3 & 86 & Good \\
You are satisfied with $\mathrm{MDR}^{\mathrm{b}}$ surveillance & 3.9 & 89 & Average \\
Average & 4.1 & 82 & Good \\
\hline
\end{tabular}

${ }^{\mathrm{a}}$ MDR-TB: multidrug-resistant tuberculosis.

${ }^{\mathrm{b}}$ MDR: multidrug resistant.

Table 6. Performance of the multidrug-resistant tuberculosis surveillance system.

\begin{tabular}{lll}
\hline Attributes & Performance & Rank \\
\hline Usefulness & Percentage score & Good \\
Flexibility & 87 & Average \\
Stability & 61 & Poor \\
Simplicity & 55 & Average \\
Acceptability & 72 & Good \\
Data quality & 82 & Good \\
Total & 91 & Average \\
\hline
\end{tabular}

\section{Discussion}

MDR-TB is a growing concern for TB programs, especially in developing countries [13]. Therefore, evaluation of MDR-TB surveillance is crucial. Our findings demonstrated good performance regarding the usefulness, especially for estimating the need for drugs to ensure case enrollment. It is also useful to ensure proper diagnosis and treatment for MDR-TB cases, which is one of the main goals for NTCP.

Based on the findings of this study, the flexibility of the system is average, as it can accommodate changes in case definitions and any updated guidelines. For example, case definition of rifampicin-resistant TB was added after GenXpert was introduced as a recommendation by the WHO [14]. Similar findings were reported from a previous evaluation in Pakistan that found that the MDR-TB surveillance can integrate with other systems [15].

Regarding the stability, we found that the MDR-TB surveillance system is unstable, mainly due to its sole dependence on donor support with a lack of governmental support. Furthermore, the frequent electricity cutoff in addition to the shortage of laboratory equipment at the regional centers negatively influenced the performance and made the system unstable, thereby affecting its sustainability. Therefore, constant power supply should be ensured for labs and appropriate laboratory equipment are essential.

The MDR-TB surveillance system seems like a simple system. Nevertheless, multiple registers and reports and the lack of a computerized system could make data collection more difficult. The acceptability of MDR-TB surveillance is good, which is reflected through the good data quality. The good acceptability might be a result of quarterly incentives for staff. However, cessation of such incentives will negatively impact acceptability and data quality. Previous similar evaluation in Pakistan found that both data quality and acceptability are good.

This study has some limitations. It did not shed light on the quantitative attributes and did not cover XDR-TB. Those limitations are attributed to the unavailability of all required lab tests for XDR-TB in addition to the specificity of MDR-TB that leads to inapplicability of some lab-related quantitative attributes. 
In conclusion, the MDR-TB surveillance system provides useful data. However, dependence on donor funds affects stability and sustainability. MDR-TB centers or units should be expanded to cover all governorates. It is also recommended that constant power supply for labs is supplied and that there is a decrease in sole dependence on donor support through gradual replacement with governmental support.

\section{Acknowledgments}

We would like to acknowledge The Eastern Mediterranean Public Health Network for their technical support.

\section{Conflicts of Interest}

None declared.

\section{References}

1. World Health Organization. Tuberculosis. URL: http://www.who.int/mediacentre/factsheets/fs104/en/ [accessed 2019-03-23]

2. World Health Organization. Multidrug-/ rifampicin-resistant TB (MDR/RR-TB):Update 2017. Tuberculosis 2017. [doi: 10.1183/1393003.congress-2017.0a1944]

3. Bastos ML, Cosme LB, Fregona G, do Prado TN, Bertolde AI, Zandonade E, et al. Treatment outcomes of MDR-tuberculosis patients in Brazil: a retrospective cohort analysis. BMC Infect Dis 2017 Nov 14;17(1):718 [FREE Full text] [doi: 10.1186/s12879-017-2810-1] [Medline: 29137626]

4. van Duin D, Paterson DL. Multidrug-Resistant Bacteria in the Community: Trends and Lessons Learned. Infect Dis Clin North Am 2016 Jun;30(2):377-390 [FREE Full text] [doi: 10.1016/j.idc.2016.02.004] [Medline: 27208764]

5. World Health Organization. 2015. Guidelines for surveillance of drug resistance in tuberculosis. URL: http://apps.who.int/ iris/bitstream/handle/10665/174897/9789241549134 eng.pdf?sequence=1 [accessed 2019-09-18]

6. World Health Organization. 2016. Global tuberculosis report. URL: https://apps.who.int/medicinedocs/documents/s23098en/ s23098en.pdf [accessed 2019-09-18]

7. World Health Organization. Strategic plan for the prevention and control of multidrug-resistant and extensively drug-resistant tuberculosis in the Eastern Mediterranean Region. 2010.

8. World Health Organization. 2016. Global Health Observatory Data Repository URL: http://gamapserver.who.int/gho/ interactive charts/tb/treatment/atlas.html [accessed 2019-09-18]

9. Yemen MOPH, Guidelines OMODRTB. NTCP. In: Guidelines on Management of Drug Resistant-TB. NTCP. Yemen: Yemen Ministry of Public Health and Population; 2012.

10. Safdar R, Khan S, Asghar R. Evaluation of hepatitis surveillance systems in Pakistan. International Journal of Infectious Diseases 2008 Dec;12:e192. [doi: 10.1016/j.ijid.2008.05.478]

11. Saeed K, Bano R, Asghar R. Evaluation of national tuberculosis surveillance system in Afghanistan. East Mediterr Health J 2013 Feb 01;19(02):200-207. [doi: 10.26719/2013.19.2.200]

12. Ansari J, Kazi B, Salman M, Asghar R. Evaluation of the existing bacterial meningitis surveillance system in Islamabad, Pakistan. International Journal of Infectious Diseases 2008 Dec;12:e192. [doi: 10.1016/j.ijid.2008.05.477]

13. Zaman K. Tuberculosis: a global health problem. J Health Popul Nutr 2010 Apr;28(2):111-113 [FREE Full text] [doi: 10.3329/jhpn.v28i2.4879] [Medline: 20411672]

14. World Health Organization. 2016. Tuberculosis, drug-resistant types URL: http://www.who.int/tb/areas-of-work/ drug-resistant-tb/types/en/ [accessed 2019-09-18]

15. Khudaidad F, Saeed A. Evaluation of drug resistant tuberculosis (DR-TB) surveillance system in Balochistan Province, in six emphenet regional conference. 2016. [doi: 10.2196/10536]
Abbreviations
DST: drug susceptibility testing
MDR: multidrug resistant
MDR-TB: multidrug-resistant tuberculosis
NTCP: National Tuberculosis Control Program
WHO: World Health Organization
XDR-TB: extensively drug-resistant tuberculosis 
Edited by H Abbas; submitted 07.04.19; peer-reviewed by A AlSaabri, F Lami; comments to author 21.05.19; revised version received 31.05.19; accepted 03.07.19; published 03.10.19

Please cite as:

Abdulmughni J, Mahyoub EM, Alaghbari AT, Al Serouri AA, Khader $Y$

Performance of Multidrug-Resistant Tuberculosis Surveillance in Yemen: Interview Study

JMIR Public Health Surveill 2019;5(4):e14294

URL: https://publichealth.jmir.org/2019/4/e14294

doi: $10.2196 / 14294$

PMID: $\underline{31584002}$

(CJihan Abdulmughni, Esam Mohammed Mahyoub, Abdulaziz Thabit Alaghbari, Abdulwahed Abdelgabar Al Serouri, Yousef Khader. Originally published in JMIR Public Health and Surveillance (http://publichealth.jmir.org), 03.10.2019 This is an open-access article distributed under the terms of the Creative Commons Attribution License (https://creativecommons.org/licenses/by/4.0/), which permits unrestricted use, distribution, and reproduction in any medium, provided the original work, first published in JMIR Public Health and Surveillance, is properly cited. The complete bibliographic information, a link to the original publication on http://publichealth.jmir.org, as well as this copyright and license information must be included. 